Article

\title{
Genome-Wide Identification of Barley ABC Genes and Their Expression in Response to Abiotic Stress Treatment
}

\author{
Ziling Zhang, Tao Tong, Yunxia Fang, Junjun Zheng, Xian Zhang, Chunyu Niu, Jia Li, \\ Xiaoqin Zhang * and Dawei Xue *(D) \\ College of Life and Environmental Sciences, Hangzhou Normal University, Hangzhou 311121, China; \\ zhangziling@stu.hznu.edu.cn (Z.Z.); tongtao@stu.hznu.edu.cn (T.T.); yxfang12@163.com (Y.F.); \\ zhengjunjun0415@163.com (J.Z.); zhangxian@hznu.edu.cn (X.Z.); niuchunyu@stu.hznu.edu.cn (C.N.); \\ lijia@stu.hznu.edu.cn (J.L.) \\ * Correspondence: xiaoqinzhang@163.com (X.Z.); dwxue@hznu.edu.cn (D.X.)
}

Received: 22 August 2020; Accepted: 27 September 2020; Published: 28 September 2020

\begin{abstract}
Adenosine triphosphate-binding cassette transporters (ABC transporters) participate in various plant growth and abiotic stress responses. In the present study, $131 \mathrm{ABC}$ genes in barley were systematically identified using bioinformatics. Based on the classification method of the family in rice, these members were classified into eight subfamilies (ABCA-ABCG, ABCI). The conserved domain, amino acid composition, physicochemical properties, chromosome distribution, and tissue expression of these genes were predicted and analyzed. The results showed that the characteristic motifs of the barley $\mathrm{ABC}$ genes were highly conserved and there were great diversities in the homology of the transmembrane domain, the number of exons, amino acid length, and the molecular weight, whereas the span of the isoelectric point was small. Tissue expression profile analysis suggested that $\mathrm{ABC}$ genes possess non-tissue specificity. Ultimately, 15 differentially expressed genes exhibited diverse expression responses to stress treatments including drought, cadmium, and salt stress, indicating that the $\mathrm{ABCB}$ and $\mathrm{ABCG}$ subfamilies function in the response to abiotic stress in barley.
\end{abstract}

Keywords: barley; $\mathrm{ABC}$ gene family; gene expression; abiotic stress

\section{Introduction}

Named after the binding frame of adenosine triphosphate (ATP), ATP-binding cassette transporters (ABC transporters) are widely found in eukaryotes and prokaryotes [1]. A previous study found that $A B C$ transporters, as one of the most widely functional protein superfamilies, are involved in plant physiological processes [2], such as plant hormone transport, nutrient uptake by organisms, stomatal regulation [3], environmental stress responses, and the interaction between plants and microorganisms [4]. Plant ABC transporters possess nucleotide-binding domains (NBDs) and transmembrane domains (TMDs), and the NBD is a hydrophilic domain with several highly conserved motifs, characterized by the Walker A and Walker B sequences, the ABC signature motif (also known as Walker C) [5], and the H loop, and the Q loop [6]. In contrast to the NBD domain, ABC proteins contain low homologous hydrophobic transmembrane domains (TMDs), and they typically consist of at least six transmembrane $\alpha$-helices. The NBD domain provides energy through combining and hydrolyzing ATP, whereas the TMD domain is able to select substrates for transportation across membranes through the energy channel provided by the former [7]. TMDs function as selectors for substrates to translocate membrane proteins through the NBD energy channel. A typical ABC full-size transporter have a core unit of two pore-forming TMDs and two cytosolic NBDs [2]. Half-size transporters, composed of only one TMD and one NBD, are thought to form homodimerize or heterodimerize that act as functional pump [8]. In many identified 
ABC transporters in bacteria, some NBDs and TMDs are present on different polypeptides, called 1/4 molecular transporters [9]. Meanwhile, a few $\mathrm{ABC}$ transporters are not directly involved in transport and have been found to participate in other cellular processes such as DNA repair and the transcription and regulation of gene expression [10-14]. In conclusion, although the amino acid sequence of $A B C$ transporters is homologous, the function of $\mathrm{ABC}$ transporters is diverse owing to their different structures.

As a consequence of the rapid development of whole-genome sequencing, the $\mathrm{ABC}$ gene family had been identified in an increasing number of plants, including 131 in Arabidopsis thaliana [15], 125 in rice, 314 in rape [16], and 130 in maize [17]. A large number of plant $A B C$ transporters are plant secondary metabolites that have evolved in response to a particular living environment. Owing to the wide range and large number of $\mathrm{ABC}$ transporters, several methods have been proposed to $\mathrm{ABC}$ protein nomenclature [18]. According to the homologous relationships, phylogenetic relationships, and domain organization, the new nomenclature system of HUGO system (Human Genome Organization) categorizes $\mathrm{ABC}$ transporters into eight subfamilies, $\mathrm{ABCA}$ to $\mathrm{ABCH}$ subfamily. However, $\mathrm{ABCH}$ has not been characterized in plants $[19,20]$. Afterwards, only ABCI subfamily containing "prokaryotic"-type ABCs has been identified in plants [18]. In total, eight subfamilies (ABCA-ABCG and $\mathrm{ABCI}$ ) have been identified in plant genomes [21].

It is well universal that abiotic stresses, such as temperature, drought, salt, and heavy metals, seriously restrict plant growth and affect the yield and quality of crops [22]. In severe cases, abiotic stress will directly result in plant death. A growing number of studies have demonstrated that $A B C$ transporters play a pivotal role in crop yield [23], quality formation [24], and the resistance response [25]. Given the importance of $A B C$ transporters in plant life activities, increasingly, plant $A B C$ transporters have been identified, cloned, and functionally analyzed [26]. To date, the ABC gene family has not been identified and analyzed in barley, and its association with abiotic stress has not been explored. Barley (Hordeum vulgare L.) is one of the oldest cultivated crops in the world. It is widely adaptable and exhibits strong drought, cold, and salt tolerance characteristics [27]. In the research, we used bioinformatics method to conduct a whole-genome study of the $A B C$ gene family in barley and identified $131 \mathrm{ABC}$ transporters. We performed sequence characteristics, physicochemical properties, gene phylogeny, and expression profile analysis of $\mathrm{ABC}$ proteins at the genomic level in barley. We also investigated the expression patterns of barley under cadmium $(\mathrm{Cd})$, drought, and salt stresses by quantitative real-time (qRT)-PCR. Our findings improve understanding of the function of the ABC gene family and will facilitate further studies on detailed molecular and biological functions in barley.

\section{Materials and Methods}

\subsection{Identification of $A B C$ Gene Family Members in Barley}

The ABCs domain-containing protein and genome sequence were retrieved from Pfam database (http://pfam.xfam.org/) [28]. The identified ABC sequences of barley and rice were confirmed for the presence of PFAM domain PF00005 (ABC transport domain) and PF00664 (ABC transmembrane domain) in barley and rice using HMMER program. In order to ensure accuracy analysis, we uploaded conserved sequences into NCBI-CDD [29] and SMART database [30] (http://smart.embl -heidelberg.de/) for protein prediction and unannotated sequences were removed. At the same time, the final protein-coding sequences were verified by searching NCBI non-redundant protein sequence database with BLASTP. Protein features including molecular weight and isoelectric point (pI) of the HvABC proteins were predicted and analyzed by using tools from ExPAsy website (https://web.expasy.org/protparam/) [31,32].

\subsection{Multiple Sequence Alignment and Phylogenetic Analysis of the ABC Gene Family in Barley}

For the sake of understanding the phylogenetic relationship of $\mathrm{ABC}$ proteins between barley and rice, the phylogenetic tree was constructed using all the identified $A B C$ amino acid sequences of barley and rice. Multiple alignments of sequences were conducted using MUSCLE [33] of the EMBL-EBI [34] 
software with the default options. Then, MUSCLE website was utilized to construct the phylogenetic tree by the neighbor-joining (NJ) method with a bootstrap test of 1000-fold (https://www.ebi.ac.uk/Tools/ $\mathrm{msa} / \mathrm{muscle} /$ ) [35]. The results were displayed using iTOL visualization.

\subsection{Analysis of ABC Gene Structure and Chromosome Location in Barley}

The information of barley $\mathrm{ABC}$ gene family, including intron, exon, physical location on chromosome and gene annotation file information, was retrieved in the Ensemble Plants database.

The exon-intron organizations of all the $H v A B C$ genes were exhibited using the online program Gene Structure Display Server (http://gsds.cbi.pku.edu.cn/) [36]. The MEME online program for protein sequence analysis was used to identify conserved motifs of $A B C$ proteins (http://meme-suite.org/t ools/meme) [37]. The MG2C was used to draw the location images of HvABCs on chromosomes. The protein sequence was used to predict subcellular localization of barley $\mathrm{ABC}$ gene family using WoLF PSORT (https://wolfpsort.hgc.jp/) [38].

\subsection{Construction of $A B C$ Gene Expression Profiles in Barley}

To create the expression profile of $H v A B C$ genes among different organs and development stages, the RNA-seq data from various tissues in barley were retrieved from IPK (https://webblast.ipk-gatersl eben.de/barley_ibsc/index.php). The dataset, 14 stages, included the, root (ROO1, ROO2), leaves (LEA, SEN), inflorescences (INF2, LOD, PAL, LEM, RAC), grain (CAR5, CAR15), etiolated seeding (ETI), tillers (NOD), and epidermal strips (EPI). The transcript abundance of $H v A B C$ genes was calculated as fragments per kilobase of exon model per million mapped reads (FPKM) and log2 (FPKM+1) values of invertase genes in these tissues were used to depict heatmaps. The cluster results were shown using the Multiple Experiment Viewer (MeV) (J. Craig Venter Institute, La Jolla, CA, USA).

\subsection{Stress Treatment, Total RNA Extraction, and qRT-PCR Analysis}

The barley cultivar, Morex, was selected for stress treatments. Seedlings were grown on nutrient solution [39] in growth chambers at $26^{\circ} \mathrm{C}$ under a $14 / 10 \mathrm{~h}$ light/dark photoperiod and photosynthetically activated radiation at 18,000 lx. Two-leaf-stage plants were treated with different abiotic stress. For drought, salt, and cadmium treatments, the seedlings were treated with 20\% PEG6000, 200 $\mathrm{mmol} \cdot \mathrm{L}^{-1} \mathrm{NaCl}$ and $50 \mu \mathrm{mol} \cdot \mathrm{L}^{-1} \mathrm{CdCl}_{2}$ nutrient solution for $24 \mathrm{~h}$, respectively. All these leaf samples were snap-frozen in liquid nitrogen and the total RNAs were isolated from young leaves using an RNA kit (AxyPrep, USA). Then, the RNA was reverse-transcribed using the HifairTM II 1stStrand cDNA Synthesis Kit following the manufacturer's instructions. RNA extraction and cDNA synthesis from all samples were stored at $-80^{\circ} \mathrm{C}$ for RNA extraction.

Based on NCBI, ABC transporters related to abiotic stress, such as rice, wheat and maize, were retrieved. Then, the phylogenetic tree with barley $A B C$ transporter (the method is the same as above) was constructed, combined with the expression information of barley $\mathrm{ABC}$ gene, expression site, and gene intron, $15 \mathrm{Hv} A B \mathrm{C}$ genes were selected to conduct qRT-PCR. cDNA obtained was used for quantitative RT-PCR using SYBR Green Master Mix and a BioRad CFX96 real-time system. The qRT-PCR experiments were performed with three biological and technical replicates. The relative expression levels were calculated using the formula $2^{-\Delta \Delta C T}$ [40]. The result was analyzed using SigmaPlot v10.0. Primers for qRT-PCR were designed using Primer Premier v5.0., using the website of Ensemble Plants to verify the specificity of primers. The barley actin gene HvActin (HORVU1Hr1G002840) was used as an internal control. The primers sequence used are listed in Table 1. 
Table 1. Primer sequence used for qRT-PCR amplification of ABC gene family in barley.

\begin{tabular}{|c|c|c|c|}
\hline Gene Name & Primer & Forward Primer Sequence $\left(5^{\prime}-3^{\prime}\right)$ & Reverse Primer Sequence $\left(5^{\prime}-3^{\prime}\right)$ \\
\hline$H v A B C G 45$ & $\mathrm{ABC1}$ & GGCGGAACTGCTGTCATCT & AGTCGGCAACACCCTTTCT \\
\hline HvABCG48 & $\mathrm{ABC} 2$ & CAGCCTGGGTTCGTTTGAG & TCGGAGTGATCGCCGTTGT \\
\hline$H v A B C G 38$ & $\mathrm{ABC} 3$ & GGTTTGGATGCTCGTGCTG & TGATTTGACCGCCTCTTTT \\
\hline$H v A B C A 3$ & $\mathrm{ABC} 4$ & TGGGCTCATTCCACCTACA & CCGTCAATGTTTCCCAGAG \\
\hline$H v A B C F 5$ & $\mathrm{ABC} 5$ & TGGCTGGAAGAAACACTGAA & TCGGGTCTGCACATACTGGTC \\
\hline$H v A B C F 4$ & ABC6 & CACATGCAGAACAAGACCCTC & GCTTCGCAGATCCATGACC \\
\hline$H v A B C C 16$ & $\mathrm{ABC7}$ & GCCATTCGGCGACCATACA & CACGAGCAAGCTGAACACG \\
\hline$H v A B C C 11$ & $\mathrm{ABC} 8$ & GTCCTTGACGCTGATACTGG & TAGCACTGGTGCCTCСТCC \\
\hline$H v A B C B 24$ & ABC9 & TGATACTGGGATTTGGTTAGG & CGAATGGCACTGAGAATGAG \\
\hline$H v A B C B 13$ & $\mathrm{ABC} 10$ & CGTTCAACTCGGAGGACAAGA & CCATGCAGCGTACCACAGG \\
\hline$H v A B C G 27$ & ABC11 & GAGGGAGGCAGCGTCAAGCA & GCAGGATGGCGAACTGGTTG \\
\hline$H v A B C G 29$ & $\mathrm{ABC} 12$ & AGGGCTTCCCGTTGTAGGTG & TCGCATCCGTCATCACCATG \\
\hline$H v A B C G 25$ & $\mathrm{ABC} 13$ & GTTCTGGATCGAGATGGGTGT & GAAGATGGTCGCCAGGATGA \\
\hline$H v A B C G 21$ & $\mathrm{ABC} 14$ & ATACCGGCATACTGGCTGTGG & CCAGCACTCGCTCCTTCACC \\
\hline$H v A B C G 18$ & $\mathrm{ABC} 15$ & TGCTCACCGCCAACTCATTC & TCCTTGCTCGCCACGAAGT \\
\hline HvActin & Actin & TGGATCGGAGGGTCCATCCT & GCACTTCCTGTGGACGATCGCTG \\
\hline
\end{tabular}

\section{Results}

\subsection{Identification and Physicochemical Properties of the ABC Gene Family in Barley}

Through multiple bioinformatics analyses, a total of $131 \mathrm{ABC}$ transporter genes were identified in barley (Supplementary Table S1). The barley $A B C$ genes were classified according to their sequence similarity with rice $A B C$ genes and were further named $H v A B C A-H v A B C G, H v A B C I$. Among the 131 HvABC proteins, all of the proteins contained one or more NBDs domains based on the domain composition analysis of the ABC proteins.

Comprehensive information on the HvABCs, including the domain structure, predicted protein length, exon number, molecular weight (MW), isoelectric point (PI), and subcellular localization, is provided in Supplementary Table S1. The amino acid numbers scoped from 171 aa (HvABCG1) to 1628 aa (HvABCC9), and the corresponding molecular weight changed from 19391.19 to 182783.15 $\mathrm{kD}$. The protein lengths of $\mathrm{ABCA}, \mathrm{ABCE}, \mathrm{ABCF}$, and $\mathrm{ABCI}$ had few differences, while the protein lengths of $A B C B, A B C C$, and $A B C G$ varied greatly. In contrast, the variation in PI was small, with the majority constituting basic proteins. Based on the subcellular localization prediction of 131 barley $A B C$ genes, $99 A B C$ genes were detected to be localized on the plasma membrane, which confirmed that most of the $\mathrm{ABC}$ transporters are bound to the plasma membrane and are responsible for the efflux of intracellular substances, while only a few are present on the vacuoles, chloroplasts, and mitochondria in these organelles. ABC transporters mainly regulate the division of exogenic substances into the organelles, which also reflect the endosymbiotic origin of the plastids and mitochondria [41]. Previous studies on the ABCC subfamily have shown that ABCCs are involved in cellular detoxification, which may contribute to the complexation of toxins and heavy metal ions with glutathione or organic acids for storage in the vacuoles or transportation out of cells [42,43]. All 22 genes of the ABCC subfamily in barley reside in the plasma membrane.

\subsection{Phylogenetic Analysis of the ABC Gene Family in Barley}

Previous studies have consistently demonstrated that the ABC gene family underwent species-specific amplification after the divergence between dicotyledonous and monocotyledonous plants [44]. Exploring the relationship between HvABC proteins and OsABC proteins could help classify and assess the potential functions of HvABCs. For further classification, ABC sequences from two different plant species, including $131 \mathrm{HvABC}$ proteins and 100 OsABC proteins, were subjected to phylogenetic analysis. Based on the phylogenetic relationships with OsABCs, the HvABCs were divided into eight subfamilies (ABCA-ABCG, ABCI) (Figure 1). In the light of phylogenetic tree, 
except $\mathrm{ABCI}$ were dispersed, the $\mathrm{HvABC}$ proteins of each subfamily were clustered together. Among those subfamilies, the ABCB and ABCG subfamily had the greatest number of members with 32 genes and 49 genes, accounting for $21.97 \%$ and $37.12 \%$, respectively. Only three members were identified in ABCE (Supplementary Table S1). Further investigation revealed that the HvABCE and HvABCF proteins contained two NBDs but, as expected, no TMD. Although their NBDs domains share sequence homology with other members of the ABC gene family in barley, they are not transposons in the conventional sense and have no obvious transport function. Analysis of the phylogenetic tree terminal branches indicated that there were 67 pairs of orthologous proteins between species, among which the ABCG and ABCB subfamily were the greatest with 23 pairs and 18 pairs, respectively, indicating that the $\mathrm{ABC}$ gene family retained very high homology in the evolution of barley and rice. In addition, there were 20 pairs of paralogs, among which the $A B C A, A B C B, A B C C$, and $A B C G$ subfamilies of barley contained one, four, three, and five pairs, respectively, and there were seven pairs of paralogs in rice; that is, the $\mathrm{ABCB}, \mathrm{ABCC}$, and $\mathrm{ABCG}$ subfamilies contained two, one, and four pairs, respectively. The above findings can infer that the members of the ABC gene family of barley may have evolved independently and expanded in a species-specific way.
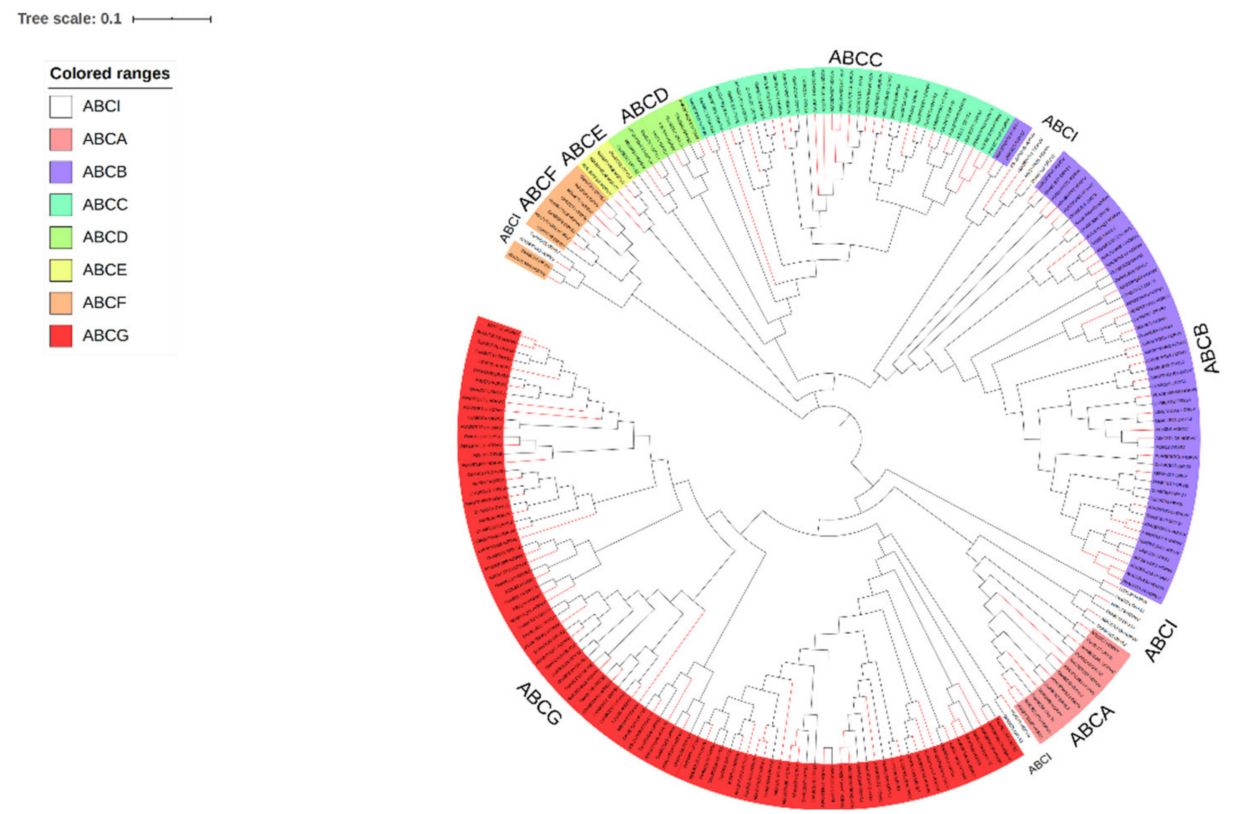

Figure 1. Unrooted Neighbor-Joining tree constructed with ABC proteins of Hordeum vulgare L. (HORVV) and Oryza sativa L. (ORYSJ). The domains clustered into eight subgroups (ABCA-ABCG, ABCI). Different colored shadings indicated eight $\mathrm{ABC}$ transporter subfamilies. The red branch is barley, and the black branch is rice.

\subsection{Chromosome Mapping of the ABC Gene Family in Barley}

A total of $131 \mathrm{HvABCs}$ were mapped on the seven chromosomes, and the remaining three genes were distributed on unanchored scaffolds (Figure 2). Chromosome mapping revealed that the $H v A B C$ genes were mostly concentrated on or near the end of the chromosomes where they exhibited a high variation in their distribution. In addition, 31 genes, the maximum number, were located on chromosome $3 \mathrm{H}$. On the contrary, chromosome $6 \mathrm{H}$ contained only nine genes. As shown in Figure 2, eight $H v A B C$ s clusters ( $H v A B C A 4 / H v A B C A 7, H v A B C B 11 / H v A B C B 16 / H v A B C B 22, H v A B C B 4 / H v A B C B 27$, $H v A B C G 29 / H v A B C B 23, H v A B C C 22 / H v A B C C 2, H v A B C C 6 / H v A B C C 8, H v A B C C 16 / H v A B C C 15 / H v A B C I 1$, and $H v A B C C 13 / H v A B C G 1)$ containing 18 genes were identified on chromosomes $1 \mathrm{H}, 3 \mathrm{H}, 4 \mathrm{H}$, and $7 \mathrm{H}$. Tandem duplication was an important recent gene duplication pattern in the expansion of HvABCs 
gene family. These may be caused by tandem repeat genes. Therefore, these gene clusters may be tandem repeat arrays.
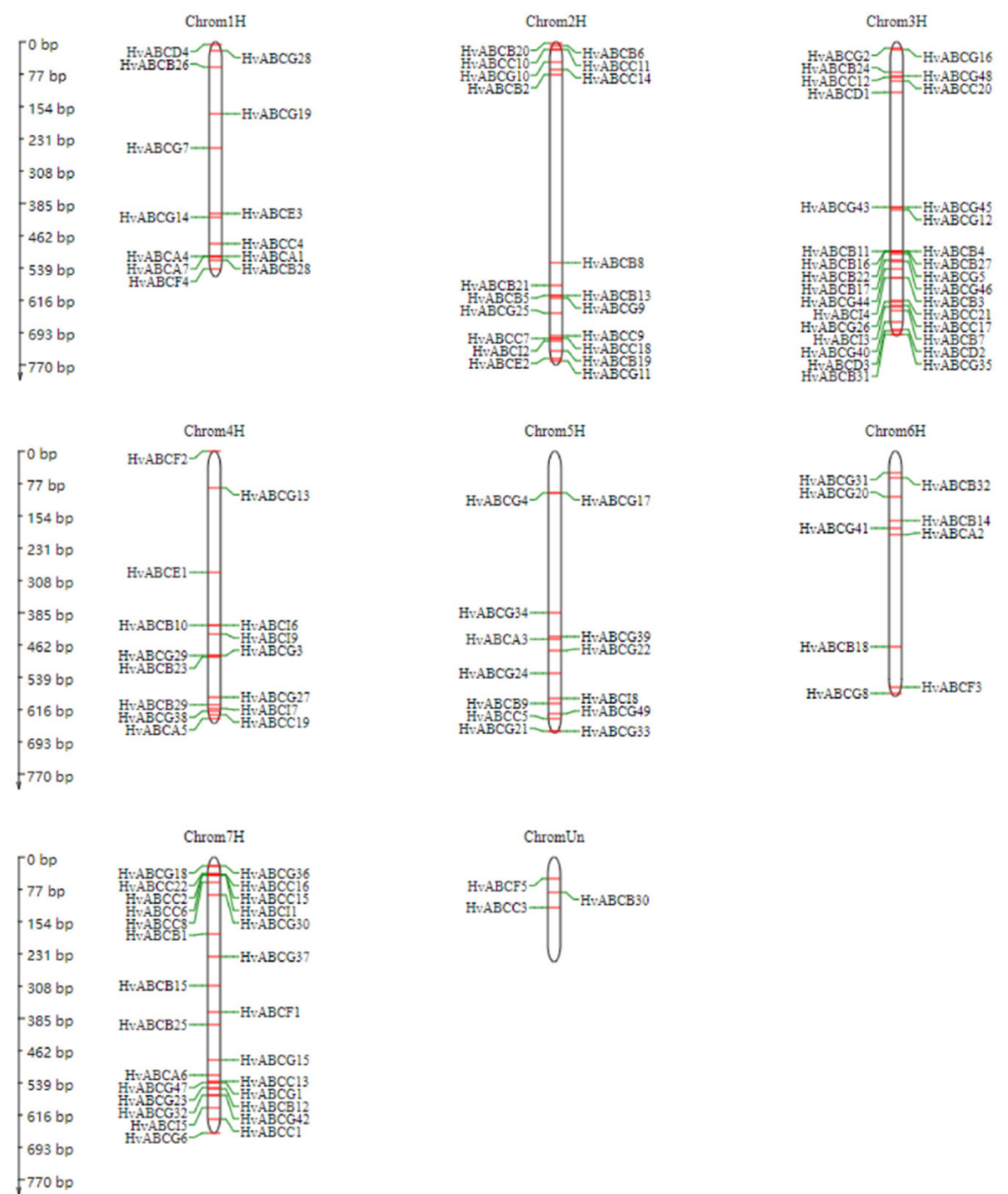

Figure 2. Mapping of the $H v A B C$ genes on Hordeum vulgare L. chromosomes. The chromosome number is indicated at the top of each chromosome. Three putative $H v A B C$ genes could not be localized on a specific chromosome.

\subsection{Analysis of Exon-Intron Structure and Conserved Domain of the ABC Gene Family in Barley}

To further explore the conservation and diversity of protein structures and compositions of HvABCs, the corresponding analysis is carried out by MEME and GSDS website. The result (Supplementary Figure S1) showed that most of the HvABC proteins (122 of 131, 93.1\%) presented multiple introns, while nine $H v A B C$ genes (HvABCG14, HvABCG15, HvABCG12, HvABCG26, HvABCG16, HvABCG28, $H v A B C G 17, H v A B C G 3, H v A B C G 1, H v A B C I 7$, and $H v A B C I 2)$ had no introns. These nine genes may have originated from the transposable events of reverse transcription. The number and size of the introns varied in the remaining 122 genes, indicating significant differences in gene structure. It can also be speculated that intron deletion and insertion events occurred in the evolutionary process, which may be caused by the differentiation of $\mathrm{ABC}$ gene family members after replication. The exons of all identified $H v A B C$ genes ranged from 1 to 30, among which the number of $A B C$ genes with 10 exons was the highest, accounting for $9.92 \%$. 
MEME motif analysis identified seven conserved motifs in the HvABC proteins. Combining the domain characteristics of each subfamily, we analyzed the conservative motifs (Supplementary Figure S2). The number of conserved motifs in each HvABC protein varies from one to five. The results (Figure 3) indicate that all seven highly conserved motifs belong to the NBD domain, which also suggests that the NBD sequence identity was higher than that of the TMDs. Among them, motif 2 is the Walker A in the nucleotide binding domains, and motif 6 is the Walker B in the nucleotide binding domains, and the motif between Walker A and Walker B is the ABC characteristic motif; that is, motif 1 , motif 3, motif 4, motif 5 , and motif 7. However, the [LIVMFY] subunit in motif 1 contains other residues (Table 2), and motif 6 in Walker B is interrupted by a hydrophilic residue. This phenomenon is currently only observed in plant ABC transporters [45]. The result also shows that several motifs are widely distributed in the HvABC proteins, such as motifs 1 and 2. In contrast, other motifs are specific to only one or two subfamilies. For instance, only $\mathrm{ABCB}$ and $\mathrm{ABCC}$ subfamilies contain motif 3 , and motif 7 exists only in $\mathrm{ABCC}$ and $\mathrm{ABCI}$. ABCG and $\mathrm{ABCF}$ contain the specific motif 4 , and these motifs are probably required for specific protein functions. The functional differentiation in HvABCs during the evolutionary process may be due to the diversity of motif components in the different subfamilies.

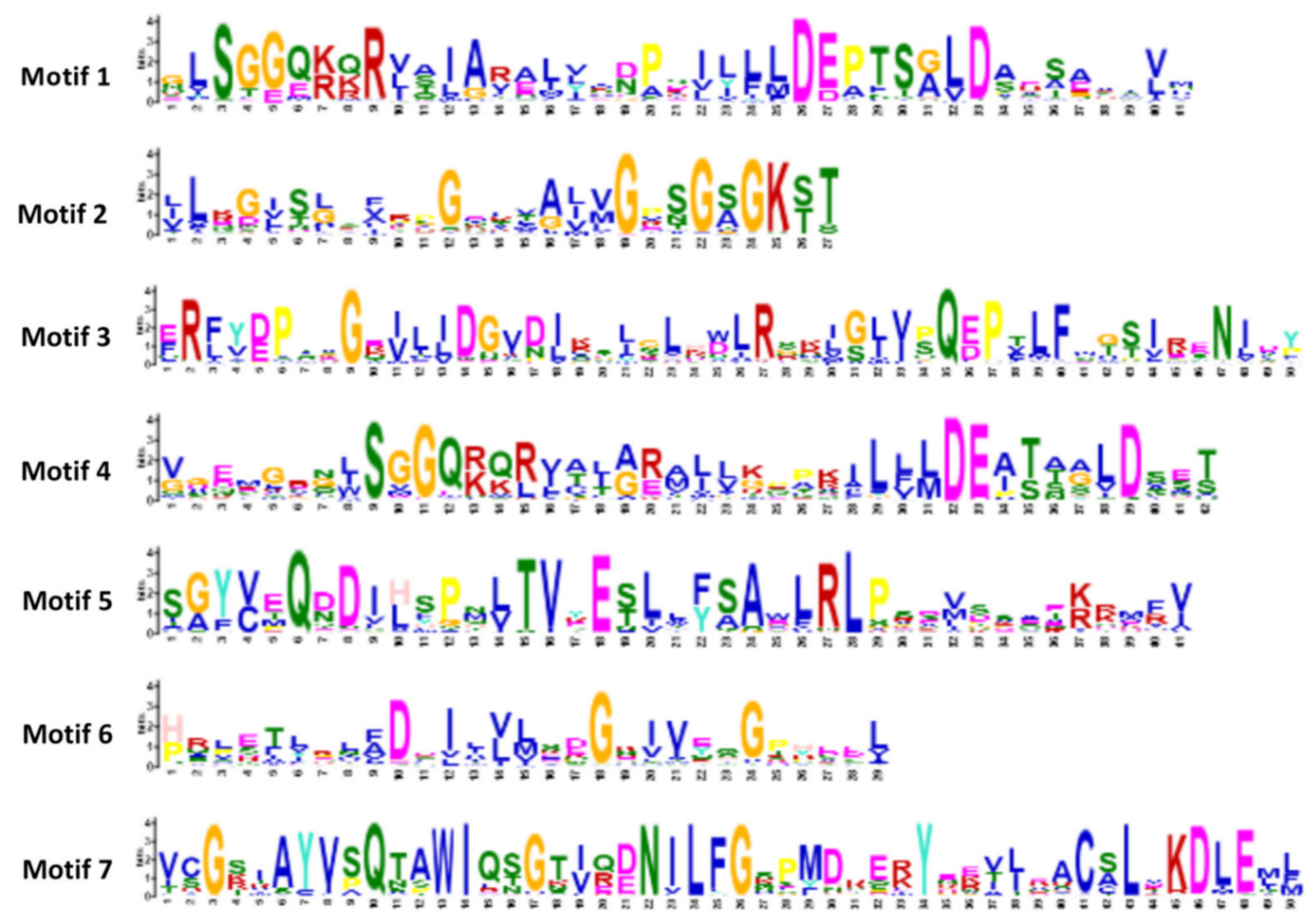

Figure 3. Conserve amino acid in seven motifs of $A B C$ gene family in barley. Motif analysis and the sequence logos was performed using MEME website.

Table 2. Seven conservative motif protein sequences given by the MEME online tool.

\begin{tabular}{cc}
\hline Motif & Protein Sequence \\
\hline Motif1 & GLSGGQKQRVAIARALLABPSILLLDEPTSGLDAESAAIVM \\
Motif2 & LLKGISLSFRPGELVALVGPSGSGKST \\
Motif3 & ERFYDPTAGEILJDGVDIKSJGLHWLRSKJGJVPQEPTLFMGSIRENIDY \\
Motif4 & VGEMGRNLSGGQRQRVALARAJLKPPKILLLDEATAALDSET \\
Motif5 & SGYVEQBDIHSPNLTVYESLLFSAWLRLPSDVSSAEKRMFV \\
Motif6 & HRLETLRLFDDILVLSDGKIVEQGPHEEL \\
Motif7 & VCGSIAYVSQTAWIQSGTIQDNILFGSPMDRERYEEVJEACSLVKDLEML \\
\hline
\end{tabular}




\subsection{Tissue-Specific Expression of ABC Genes in Barley}

Tissue-specific expression patterns of genes can help elucidate their function in plant species and predict their role in growth and development. To further elucidate the expression profiles of $H v A B C s$ in different tissues and developmental stages, we used expression data of different tissues from the IPK website as a resource. A heatmap displaying the expression data of $H v A B C s$ in different periods as well as in the tissues and organs was generated, and the $H v A B C$ s were clustered by their expression patterns. Tissue specific expression profiles showed that the $H v A B C$ genes could be categorized into eight types (Figure 4), which indicated that the expression of $A B C$ genes had undergone significant differentiation. Furthermore, the expression data clustering results did not clearly correspond to the subfamilies differentiated by the phylogenetic analysis, indicating that sequence similarity does not completely determine expression pattern and function similarity.

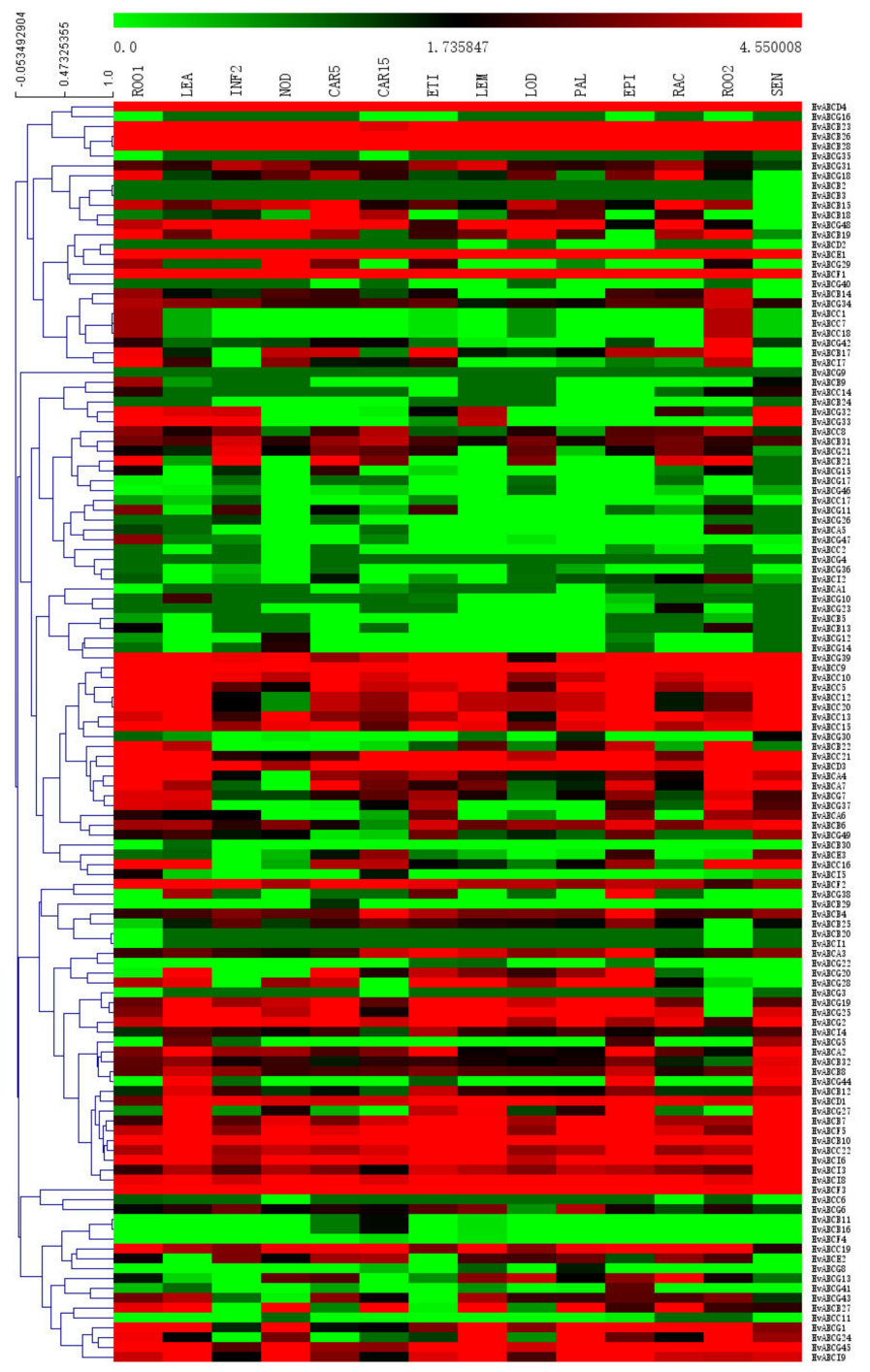

Figure 4. Heatmap showing the expression pattern of $H v A B C$ genes in developmental stages and tissues, including ROO1 (Roots from seedings), ROO2 (Roots), LEA (Shoots from seedings), ETI (Etiolated seeding, dark cond), INF2 (Developing inflorescences), PAL (Dissected inflorescences), LEM/LOD/RAC (inflorescences, lemma/lodicule/rachis), NOD (Developing tillers), CAR5/CAR15 (Developing grain, 5 DAP/15 DAP), EPI (Epidermal strips), SEN (Senescing leaves). The combined phylogenetic trees of $H v A B C$ s genes on the left panel. The scale bar at the top represents relative expression value. Red denotes high expression levels, and green denotes low expression levels. 
In this study, a total of $127 \mathrm{HvABC}$ s were determined as being expressed in at least one tissue (the FPKM values of $H v A B C B 1, H v A B C C 3, H v A B C C 4$, and $H v A B C G 9$ in the 15 tissues were all 0), and $92 \mathrm{HvABCs}$ were expressed in all tissues. The tissue-specific expression profile showed that some HvABC gene family members did not have tissue specificity and highlighted an essential role in almost all growth and developmental stages. As the Figure 4 shows, the majority of $H v A B C s$ presented different expression patterns, whereas a few exhibited similar expression patterns. Some $A B C C$ subfamily genes were ubiquitously and highly expressed in all the tissues, especially the root, such as $H v A B C C 1, H v A B C C 7$, and $H v A B C C 18$. Some $H v A B C s$ also exhibited tissue-specific expression; for instance, $H v A B C G 10$ only specifically expressed in the leaf tissues, $H v A B C B 11$ and $H v A B C B 16$ specifically expressed in the developing grain, and HvABCG12, HvABCG14, and HvABCG29 were high during tiller development, implying that these genes may play specific roles in the relevant tissues.

\subsection{Expression Analysis of ABC Genes in Barley in Response to Abiotic Stress}

Research has found that plant $\mathrm{ABC}$ transporters play a major role in auxin, heavy metal transport, and abiotic stress, especially the ABCG subfamily and $A B C B$ subfamily [46]. In this study, a total of 15 $H v A B C$ genes were identified using bioinformatics methods, including one $H v A B C A$, two $H v A B C B s$, two $H v A B C C s$, two $H v A B C F s$, and eight $H v A B C G s$. The expression responses of selected $H v A B C$ genes under $\mathrm{NaCl}, \mathrm{PEG}$, and $\mathrm{Cd}$ treatment conditions were examined using qRT-PCR in our study.

A range of expression levels were observed in the selected $H v A B C s$ at $24 \mathrm{~h}$ after exposure to $\mathrm{Cd}$, drought, and salt stress. The analyses revealed that different members within each HvABC subfamily responded differently to the same set of abiotic stresses. Compared with the control levels, the results showed that the expression levels of 13 of the 15 identified $H v A B C$ genes increased in response to Cd stress. The most pronounced increases were observed in $H v A B C A 3$ and $H v A B C G 29$, whereas $H v A B C G 38$ and $H v A B C G 21$ were dramatically repressed by $\mathrm{Cd}$ stress (Figure $5 \mathrm{~A}$ ). Under salt stress conditions, most $H v A B C$ genes exhibited the opposite pattern to Cd stress, with $H v A B C G 48$, $H v A B C F 4, H v A B C B 24$, and $H v A B C G 25$ being repressed (Figure 5B). Expression analysis following PEG treatment indicated that $A B C$ genes were upregulated by 1.0 times, including $H v A B C G 45$, $H v A B C G 48, H v A B C A 3, H v A B C F 5, H v A B C F 4, H v A B C C 16, H v A B C C 11$, and HvABCB13. Under different abiotic stresses, $H v A B C$ genes showed different response patterns and different response degrees. Among the $15 H v A B C$ genes, $H v A B C G 38$ was repressed after all the stress treatments, whereas the expression of other genes, including $H v A B C G 45, H v A B C A 3, H v A B C F 5, H v A B C C 16, H v A B C C 11$, $H v A B C B 13, H v A B C G 27, H v A B C G 29$, and $H v A B C G 18$, was increased by the stress treatments (Figure 5C). Conversely, the expressions of $H v A B C G 4, H v A B C G 25, H v A B C G 21, H v A B C F 4$, and HvABCB24 were enhanced or induced by the stress treatments. 

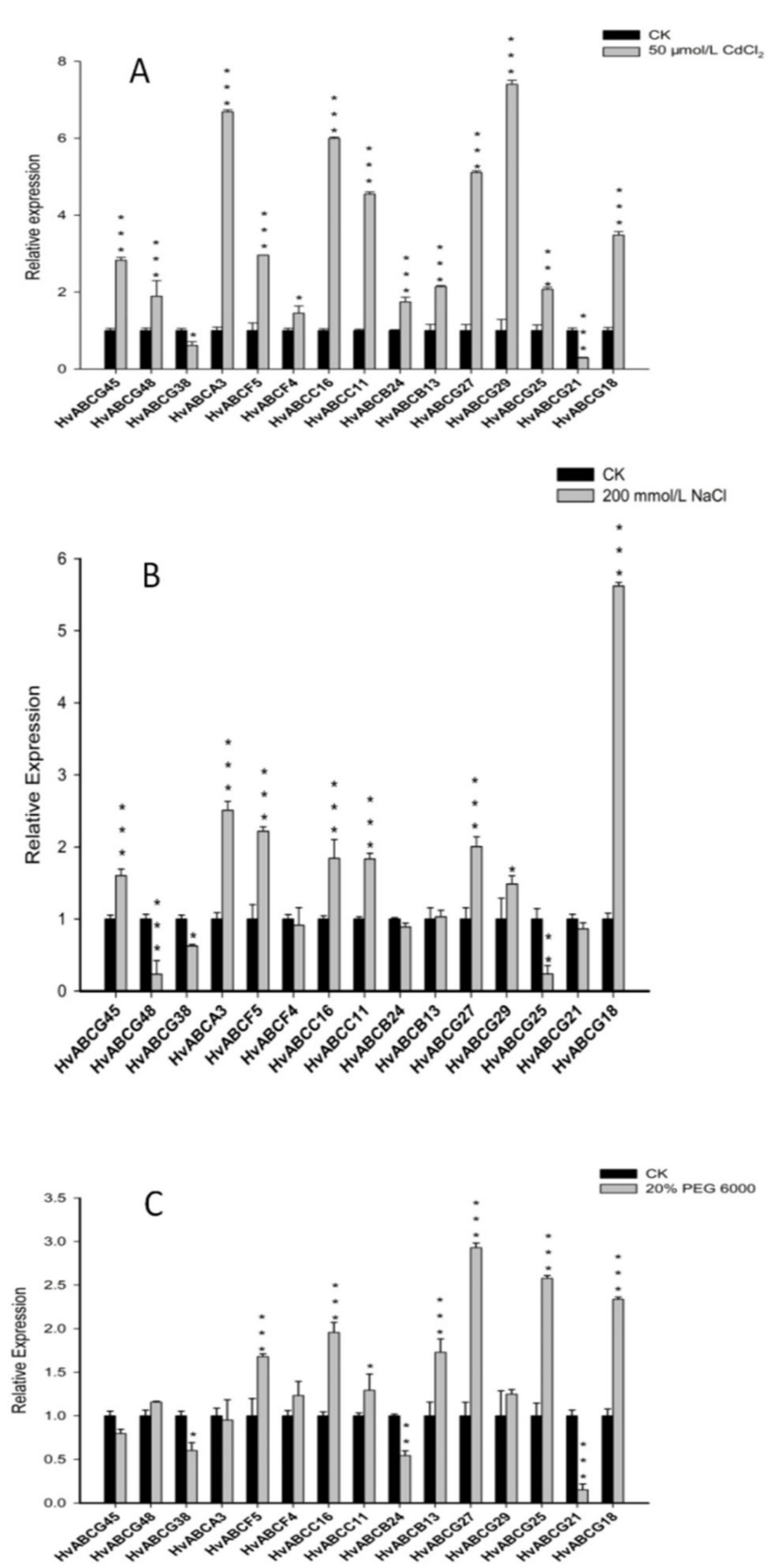

Figure 5. qRT-PCR analysis of $15 \mathrm{Hv} A B C$ genes in response to (A) $50 \mu \mathrm{mol} \cdot \mathrm{L}^{-1} \mathrm{CdCl}_{2}$ (B) $200 \mathrm{mmol} \cdot \mathrm{L}^{-1}$ $\mathrm{NaCl}$ (C) 20\% PEG6000. columns in black represent CK, columns in gray represent abiotic stress. ANOVA and LSD was used to test significance. Asterisks indicate the corresponding gene significantly up- or down-regulated compared with the untreated control $\left({ }^{*} p<0.05,{ }^{* *} p<0.01,{ }^{* * *} p<0.001\right)$ Data are the means of three replicates with standard errors represented by bars.

\section{Discussion}

The ATP-binding cassette (ABC) transporters belong to a large superfamily of proteins, which are ubiquitous and important in all kind of life events for all life organism $[9,47]$. In plants, ABC transporters participate in the transport of exogenous substances and secondary metabolites and in the abiotic stress response, as well as in many other important physiological and developmental processes $[6,21]$.

Because of repeated genome replication, the number of $\mathrm{ABC}$ proteins in plants is much higher than in animals. Given the important regulatory role of $\mathrm{ABC}$ transporters in plant growth and development, bioinformatics analysis of $\mathrm{ABC}$ family genes has been conducted in Arabidopsis [15], rice [48], maize [17], 
Lotus corniculatus [49], grape [50], Brassica napus [16], and other important plants. Thus far, most research into the functions of $\mathrm{ABC}$ transporters has been concentrated on Arabidopsis. In this study, 131 HvABC proteins were identified genome-wide in barley (Supplementary Table S1), which is similar to the number of genes identified in rice and Arabidopsis [15,48]. Additionally, phylogenetic analysis divided the protein sequences of barley and rice into eight subfamilies, including ABCA-ABCG and $A B C I$ (Figure 1). In each subfamily, the $A B C$ orthologous genes reflect the sequence similarity of barley and rice, and relatively, the emergence of paralogous genes indicates differentiation It is presumed that the barley genome experienced whole-genome replication events before the separation of gramineous species. All $H v A B C$ genes have at least one NBD domain, which indicates that NBD is a unique domain of $\mathrm{ABC}$ transporters. The HvABC transporter domain has various organizational forms that can be split into full-size members, half-sized transporters, and 1/4 molecular transporters [8,9]. $H v A B C$ include multi-domains, whereas few genes generally contain only one NBD domain. This phenomenon has been confirmed in $S L A B C B 27$ of the tomato ABC gene family [51]. Conserved sequence analysis also reflects the uniqueness of the $A B C$ domain and the high conservation of NBDs. On the contrary, the sequences and structures of TMDs differ, which reflects the chemical diversity of the substrates transported by $\mathrm{ABC}$ transporters. The members of the HvABC gene family have large functional differences, with large span amino acid, as well as large differences in isoelectric point and $\mathrm{pH}$ value (Supplementary Table S1). In short, the structural differences also reflect the different functions.

Gene expression pattern is an important starting point for further evolution and function research. Thus, we conducted expression analysis for all HvABCs in 15 tissues and organs. Except for a few genes, most HvABCs did not exhibit clear tissue specificity (Figure 4), indicating that HvABCs play a role in all aspects of plant growth. Studies have shown that Arabidopsis AtABCB19 participates in the regulation of the separation of post-embryonic organs and cytoplasmic flow in the inflorescence axis of Arabidopsis [52,53], while AtABCB1 plays a major role in another development, and AtABCB19 plays a synergistic role [54]. The AtABCG11 protein is distributed in the stems, leaves, and floral organs of plants and is involved in the transportation of paraffin wax and grease substances on the surface of plants.

Previous studies have found that the ABCB subfamily may be closely associated with abiotic stress in plants $[17,55,56]$. For example, AtABCB25 is related to heavy metal resistance in Arabidopsis, and the overexpression of $A t A B C B 25$ can improve the resistance of Arabidopsis to Cd and lead [57]. OsABCB23 and $O s A B C B 24$ are induced by drought stress, while $O s A B C B 6, O s A B C B 9$, and $O s A B C B 8$ are induced by salt stress $[48,58]$. OsABCB27, located on the vacuolar membrane, participates in the response of rice to aluminum stress [59]. Under drought stress, the expression of the $Z m A B C B 7$ and $Z m A B C B 8$ genes increases significantly, while the expression of $Z m A B C B 18$ is significantly inhibited by salt stress [17]. In this study, it was found that the expression of $H v A B C B 13$ was significantly increased under $\mathrm{Cd}$, drought, and salt stress, while $H v A B C B 24$ was inhibited by salt stress and drought, but could respond significantly to $C d$ stress. ABCG transporters excrete wax and keratinous monomers, thus reducing water loss [60]. AtABCG12 participates in the transmembrane transport of cuticular wax in the stem epidermis, AtABCG32 is involved in the formation of the cell wall cuticle, and the AtABCG32 transporter substrate is a keratinous monomer. When plants suffer from drought stress, AtABCG40 increases abscisic acid production to timeously close the stomata [61]. At the same time, as the largest subfamily, ABCG also plays an important role in abiotic stress. SpTUR2 in duckweed is expressed upon exposure to salicylic acid, cold, and high-salt environments [62]. The overexpression of the AtABCG36 gene can improve the resistance of Arabidopsis to salt and drought [63]. The ABCG transporter OsABCG9 in rice roots responds significantly to PEG, zinc, and Cd stress, and its expression is closely related to redox mechanism and heavy metal stress [64]. Our study shows that the HvABCG subfamily responded to salt, drought, and Cd stress to different degrees. HvABCG45, HvABCG27, $H v A B C G 29$, and $H v A B C G 18$ were concurrently involved in three types of stress induction. In addition, $H v A B C G 38$ and $H v A B C G 21$ could significantly respond to salt stress, and their expression levels decreased significantly after drought and Cd treatments. HvABCG48 and HvABCG25 were significantly 
upregulated under drought and $\mathrm{Cd}$ stress but were inhibited by salt stress (Figure 5). This also confirms that the HvABCB and HvABCG subfamily are involved in the abiotic stress response, and most HvABC genes are regulated by at least one abiotic stress factor.

A genome-wide analysis of ABC gene family in barley was carried out in the present study. The phylogenetic relationships, gene structures, chromosome locations, and expression profiles of $H v A B C$ s were studied in detail. Furthermore, 15 genes responding to abiotic stress were preliminarily screened, and the expression levels of the genes following stress treatment were analyzed. Taken together, our study revealed the functional diversity of HvABCs proteins and provided candidate $A B C$ genes for future breeding to various stresses.

Supplementary Materials: The following are available online at http://www.mdpi.com/2223-7747/9/10/1281/s1, Figure S1. Intron-exon structures of the ABC genes in barley. Analysis of HvABC genes structure using GSDS online tool. Yellow boxes indicate the coding sequences (CDS), blue rectangles indicate UTR, while black lines indicate introns. Figure S2. The motif analysis of ABC family in barley. Seven conservative motifs were identified and were represented by different colors. The black solid line represents the corresponding HvABC protein and its length. The position of the motif on the sequence was labeled. Table S1: Physicochemical properties, domain and subcellular localization of $\mathrm{ABC}$ proteins in barley.

Author Contributions: D.X., X.Z. (Xiaoqin Zhang), and Y.F. managed this project; Z.Z., T.T., and X.Z. (Xian Zhang) analyzed the sequencing data; Z.Z., J.Z., C.N., and J.L. performed the experiments; Z.Z., T.T., and D.X. wrote the manuscript. All authors have read and agreed to the published version of the manuscript.

Funding: This research was supported by the National Natural Science Foundation of China (31401316) and Hangzhou Scientific and Technological Program (20140432B03).

Acknowledgments: We thank LetPub (www.letpub.com) for its linguistic assistance during the preparation of this manuscript.

Conflicts of Interest: The authors declare that they have no competing interests.

\section{References}

1. Higgins, C.F.; Linton, K.J. The ATP switch model for ABC transporters. Nat. Struct. Mol. Biol. 2004, 11, 918-926. [CrossRef] [PubMed]

2. Davidson, A.L.; Dassa, E.; Orelle, C.; Chen, J. Structure, function, and evolution of bacterial ATP-binding cassette systems. Microbiol. Mol. Biol. Rev. 2008, 72,317-364. [CrossRef] [PubMed]

3. Lane, T.S.; Rempe, C.S.; Davitt, J.; Staton, M.E.; Peng, Y.; Soltis, D.E.; Melkonian, M.; Deyholos, M.; Leebens-Mack, J.H.; Chase, M. Diversity of ABC transporter genes across the plant kingdom and their potential utility in biotechnology. BMC Biotechnol. 2017, 16, 47. [CrossRef] [PubMed]

4. Liu, Y.Q.; Zhao, Y.F. Structure and mechanism of ABC transporter. Chin. Bull. Life Sci. 2017, 29, $223-229$.

5. Jasinski, M.; Ducos, E.; Martinoia, E.; Boutry, M. The ATP-binding cassette transporters: Structure, function and gene family comparison between rice and Arabidopsis. Plant Physiol. 2003, 131, 1169. [CrossRef]

6. Rea, P.A. Plant ATP-binding cassette transporters. Annu. Rev. Plant Biol. 2007, 58, 347-375. [CrossRef]

7. Schneider, E.; Hunke, S. ATP-binding-cassette (ABC) transport systems: Functional and structural aspects of the ATP-hydrolyzing subunits/domains. FEMS Microbiol. Rev. 1998, 22, 1-20. [CrossRef]

8. Mcfarlane, H.E.; Shin, J.J.H.; Bird, D.A.; Samuels, A.L. Arabidopsis ABCG transporters, which are required for export of diverse cuticular lipids, dimerize in different combinations. Plant Cell 2010, 22, 3066-3075. [CrossRef]

9. Higgins, C.F. ABC transporters: From microorganisms to man. Annu. Rev. Cell Biol. 1992, 8, 67-113. [CrossRef]

10. Dong, J.; Lai, R.; Jennings, J.L.; Link, A.J.; Hinnebusch, A.G. The novel ATP-binding cassette protein ARB1 is a shuttling factor that stimulates 40s and 60s ribosome biogenesis. Mol. Cell. Biol. 2005, 25, 9859-9873. [CrossRef]

11. Dong, J.; Lai, R.; Nielsen, K.; Fekete, C.A.; Qiu, H.; Hinnebusch, A.G. The essential ATP-binding cassette protein RLI1 functions in translation by promoting preinitiation complex assembly. J. Biol. Chem. 2004, 279, 42157. [CrossRef] [PubMed]

12. Garcia, O.; Bouige, P.; Forestier, C.; Dassa, E. Inventory and comparative analysis of rice and Arabidopsis ATP-binding cassette (ABC) systems. Mol. Biol. 2004, 343, 249-265. [CrossRef] [PubMed] 
13. Kärblane, K.; Gerassimenko, J.; Nigul, L.; Piirsoo, A.; Smialowska, A.; Vinkel, K.; Kylsten, P.; Ekwall, K.; Swoboda, P.; Truve, E.; et al. ABCE1 is a highly conserved RNA silencing suppressor. PLoS ONE 2015, 10, e0116702. [CrossRef] [PubMed]

14. Kovalchuk, A.; Driessen, A.J. Phylogenetic analysis of fungal ABC transporters. BMC Genomics 2010, 11, 177. [CrossRef] [PubMed]

15. Sanchez-Fernandez, R.; Davies, T.G.; Coleman, J.O.; Rea, P.A. The Arabidopsis thaliana ABC protein superfamily, a complete inventory. J. Biol. Chem. 2001, 276, 30231-30244. [CrossRef]

16. Zhang, X.D.; Zhao, K.X.; Yang, Z.M. Identification of genomic ATP binding cassette (ABC) transporter genes and Cd-responsive ABCs in Brassica napus. Gene 2018, 664, 139-151. [CrossRef]

17. Pang, K.; Li, Y.; Liu, M.; Meng, Z.; Yu, Y. Inventory and general analysis of the ATP-binding cassette (ABC) gene superfamily in maize (Zea mays L.). Gene 2013, 526, 411-428. [CrossRef]

18. Verrier, P.J.; Bird, D.; Burla, B.; Dassa, E.; Forestier, C.; Geisler, M.; Klein, M.; Kolukisaoglu, U.; Lee, Y.; Martinoia, E.; et al. Plant ABC proteins-a unified nomenclature and updated inventory. Trends Plant Sci. 2008, 13, 151-159. [CrossRef]

19. Annilo, T.; Chen, Z.Q.; Shulenin, S.; Costantino, J.; Thomas, L.; Lou, H.; Stefanov, S.; Dean, M. Evolution of the vertebrate ABC gene family: Analysis of gene birth and death. Genomics 2006, 88, 1-11. [CrossRef]

20. Dermauw, W.; Van Leeuwen, T. The ABC gene family in arthropods: Comparative genomics and role in insecticide transport and resistance. Insect. Biochem. Mol. Biol. 2014, 45, 89-110. [CrossRef]

21. Kang, J.; Park, J.; Choi, H.; Burla, B.; Kretzschmar, T.; Lee, Y.; Martinoia, E. Plant ABC Transporters. Arabidopsis Book 2011, 9, e0153. [CrossRef] [PubMed]

22. Roy, S.J.; Tucker, E.J.; Tester, M.A. Genetic analysis of abiotic stress tolerance in crops. Curr. Opin. Plant Biol. 2011, 14, 232-239. [CrossRef] [PubMed]

23. Orsi, C.H.; Tanksley, S.D. Natural variation in an ABC transporter gene associated with seed size evolution in tomato species. PLoS Genet. 2009, 5, e1000347. [CrossRef] [PubMed]

24. Xu, X.H.; Zhao, H.J.; Liu, Q.L.; Frank, T.; Engel, K.H.; An, G.; Shu, Q.Y. Mutations of the multi-drug resistance-associated protein $\mathrm{ABC}$ transporter gene 5 result in reduction of phytic acid in rice seeds. Theor. Appl. Genet. 2009, 119, 75-83. [CrossRef]

25. Crouzet, J.; Trombik, T.; Fraysse, A.S.; Boutry, M. Organization and function of the plant pleiotropic drug resistance ABC transporter family. FEBS Lett. 2006, 580, 1123-1130. [CrossRef]

26. Hwang, J.U.; Song, W.Y.; Hong, D.; Ko, D.; Yamaoka, Y.; Jang, S.; Yim, S. Plant ABC transporters enable many unique aspects of a terrestrial plant's lifestyle. Mol. Plant 2016, 9, 338-355. [CrossRef]

27. Tong, T.; Fang, Y.X.; Zhang, Z.L.; Zheng, J.J.; Xue, D.W. Genome-wide identification, phylogenetic and expression analysis of SBP-box gene family in barley (Hordeum vulgare L.). Plant Growth Regul. 2020, 90, 1-13. [CrossRef]

28. Finn, R.D.; Tate, J.; Mistry, J.; Coggill, P.C.; Sammut, S.J.; Hotz, H.R.; Ceric, G.; Forslund, K.; Eddy, S.R.; Sonnhammer, E.L.; et al. The Pfam protein families database. Nucleic Acids Res. 2008, 36, D281-D288. [CrossRef]

29. Marchler-Bauer, A.; Derbyshire, M.K.; Gonzales, N.R.; Lu, S.; Chitsaz, F.; Geer, L.Y.; Geer, R.C.; He, J.; Gwadz, M.; Hurwitz, D.I.; et al. CDD: NCBI's conserved domain database. Nucleic Acids Res. 2015, 43, D222-D226. [CrossRef]

30. Letunic, I.; Doerks, T.; Bork, P. SMART 7: Recent updates to the protein domain annotation resource. Nucleic Acids Res. 2012, 40, D302-D305. [CrossRef]

31. Artimo, P.; Jonnalagedda, M.; Arnold, K.; Baratin, D.; Csardi, G.; de Castro, E.; Duvaud, S.; Flegel, V.; Fortier, A.; Gasteiger, E.; et al. ExPASy: SIB bioinformatics resource portal. Nucleic Acids Res. 2012, 40, W597-W603. [CrossRef] [PubMed]

32. Liu, M.Y.; Sun, W.J.; Ma, Z.T. Genome-wide investigation of the AP2/ERF gene family in tartary buckwheat (Fagopyum Tataricum). BMC Plant Biol. 2019, 19, 84. [CrossRef] [PubMed]

33. Edgar, R.C. MUSCLE: Multiple sequence alignment with high accuracy and high throughput. Nucleic Acids Res. 2004, 5, 1792-1797. [CrossRef]

34. Mcwilliam, H.; Li, W.; Uludag, M. Analysis Tool Web Services from the EMBL-EBI. Nucleic Acids Res. 2013, 41, W597-W600. [CrossRef] [PubMed]

35. Edgar, R.C. MUSCLE: A multiple sequence alignment method with reduced time and space complexity. BMC Bioinform. 2004, 5, 113. [CrossRef] [PubMed] 
36. Hu, B.; Jin, J.; Guo, A.Y.; Zhang, H.; Gao, G. GSDS 2.0: An upgraded gene feature visualization server. Bioinformatics 2014, 31, 1296-1297. [CrossRef]

37. Bailey, T.L.; Boden, M.; Buske, F.A.; Frith, M.; Grant, C.E.; Clementi, L.; Ren, J.; Li, W.W.; Noble, W.S. MEME SUITE: Tools for motif discovery and searching. Nucleic Acids Res. 2009, 37, W202-W208. [CrossRef]

38. Horton, P.; Park, K.J.; Obayashi, T.; Nakai, K. Protein subcellular localization prediction with WOLF PSORT. In Proceedings of the 4th Asia-Pacific Bioinformatics Conference, Taipei, Taiwan, 13-16 February 2006.

39. Zhang, X.; Tong, T.; Tian, B.; Fang, Y.; Xue, D. Physiological, biochemical and molecular responses of barley seedlings to Aluminum stress. Phyton 2019, 88, 253-260. [CrossRef]

40. Tong, C.; Wang, X.; Yu, J.; Wu, J.; Liu, S. Comprehensive analysis of RNA-seq data reveals the complexity of the transcriptome in Brassica rapa. BMC Genomics 2013, 14, 689. [CrossRef]

41. Theodoulou, F.L. Plant ABC Transporters. Biochim. Biophys. Acta 2000, 1465, 79-103. [CrossRef]

42. Song, W.Y.; Mendoza-Cózatl, D.G.; Lee, Y.; Schroeder, J.I.; Ahn, S.N.; Lee, H.S.; Wicker, T.; Martinoia, E. Phytochelatin-metal(loid) transport into vacuoles shows different substrate preferences in barley and Arabidopsis. Plant Cell Environ. 2013, 37, 1192-1201. [CrossRef] [PubMed]

43. Sousa, C.A.; Hanselaer, S.; Soares, E.V. ABCC subfamily vacuolar transporters are involved in $\mathrm{Pb}$ (Lead) detoxification in Saccharomyces cerevisiae. Appl. Biochem. Biotechnol. 2014, 175, 65-74. [CrossRef] [PubMed]

44. Theodoulou, F.L.; Kerr, I.D. ABC transporter research: Going strong 40 years on. Biochem. Soc. Trans. 2015, 43, 1033-1040. [CrossRef] [PubMed]

45. Martinoia, E.; Klein, M.; Geisler, M.; Bovet, L.; Forestier, C.; Kolukisaoglu, U.; Müller-Röber, B.; Schulz, B. Multifunctionality of plant ABC transporters-More than just detoxifiers. Planta 2002, 214, 345-355. [CrossRef]

46. Martin, C.; Berridge, G.; Mistry, P.; Higgins, C.; Charlton, P.; Callaghan, R. Drug binding sites on P-glycoprotein are altered by ATP binding prior to nucleotide hydrolysis. Biochemistry 2000, 39, 11901-11906. [CrossRef]

47. Broehan, G.; Kroeger, T.; Lorenzen, M.; Merzendorfer, H. Functional analysis of the ATP-binding cassette (ABC) transporter gene family of Tribolium castaneum. BMC Genomics 2013, 14, 1-18. [CrossRef]

48. Xu, X.; Qiu, J.; Xu, Y.; Xu, C.W. Molecular evolution and expression analysis of subfamily ABCB transporter genes in rice. Chin. J. Rice Sci. 2012, 26, 127-136.

49. Akifumi, S.; Nobukazu, S.; Shusei, S.; Yasukazu, N.; Satoshi, T.; Kazufumi, Y. Genome-wide analysis of ATP-binding cassette (ABC) proteins in a model legume plant, Lotus japonicus: Comparison with Arabidopsis ABC protein family. DNA Res. 2006, 13, 205-228.

50. Çakır, B.; Kılıçkaya, O. Whole-genome survey of the putative ATP-binding cassette transporter family genes in Vitis vinifera. PLoS ONE 2013, 8, e78860. [CrossRef]

51. Amoako, O.P.; Ayaka, M.; Mami, S.; Enrico, M.; Stefan, R.; Koh, A.; Daisuke, S.; Shungo, O.; Shogo, M.; Katsuhiro, S. Genome-wide analysis of ATP binding cassette (ABC) transporters in tomato. PLoS ONE 2018, 13, e0200854.

52. Okamoto, K.; Ueda, H.; Shimada, T.; Tamura, K.; Hara-Nishimura, I. An ABC transporter B family protein, $\mathrm{ABCB} 19$, is required for cytoplasmic streaming and gravitropism of the inflorescence stems. Plant Signal Behav. 2016, 11, e1010947. [CrossRef] [PubMed]

53. Zhao, H.; Liu, L.; Mo, H.; Qian, L.; Cao, Y.; Cui, S.; Li, X.; Ma, L.; Merks, R.M.H.J.P.O. The ATP-Binding cassette transporter ABCB19 regulates postembryonic organ separation in Arabidopsis. PLoS ONE 2013, 8, e60809. [CrossRef] [PubMed]

54. Cecchetti, V.; Brunetti, P.; Napoli, N.; Fattorini, L.; Altamura, M.M.; Costantino, P.; Cardarelli, M. ABCB1 and ABCB19 auxin transporters have synergistic effects on early and late Arabidopsis anther development. J. Integr. Plant Biol. 2015, 57, 1089-1098. [CrossRef] [PubMed]

55. Chai, C.; Subudhi, P.K. Comprehensive analysis and expression profiling of the OsLAX and OsABCB auxin transporter gene families in Rice (Oryza sativa) under phytohormone stimuli and abiotic stresses. Front. Plant Sci. 2016, 3, 593. [CrossRef]

56. Shen, C.J.; Bai, Y.H.; Wang, B.J.; Zhang, S.N.; Wu, Y.R.; Chen, M.; Jiang, D.A.; Qi, Y.H. Expression profile of PIN, AUX/LAX and PGP auxin transporter gene families in Sorghum bicolor under phytohormone and abiotic stress. FEBS J. 2010, 277, 2954-2969. [CrossRef]

57. Kim, D.Y.; Bovet, L.; Kushnir, S.; Noh, E.W.; Martinoia, E.; Lee, Y. AtATM3 is involved in heavy metal resistance in Arabidopsis. Physiol. Plant. 2006, 140, 922-932. [CrossRef] 
58. Jayita, S.; Atreyee, S.; Kamala, G.; Bhaskar, G. Molecular phylogenetic study and expression analysis of ATP-binding cassette transporter gene family in Oryza sativa in response to salt stress. Comput. Biol. Chem. 2015, 54, 18-32.

59. Huang, C.F.; Yamaji, N.; Mitani, N.; Yano, M.; Nagamura, Y.; Ma, J.F. A bacterial-type ABC transporter is involved in aluminum tolerance in rice. Plant Cell 2009, 21, 655-667. [CrossRef]

60. Bird, D.A. The role of ABC transporters in cuticular lipid secretion. Plant Sci. 2008, 174, 563-569. [CrossRef]

61. Pighin, J.A.; Zheng, H.; Balakshin, L.J.; Goodman, I.P.; Western, T.L.; Jetter, R.; Kunst, L.; Samuels, A.L. Plant cuticular lipid export requires an ABC transporter. Science 2004, 306, 702-704. [CrossRef]

62. Von, R.L.E.; Degenkolb, T.; Zerjeski, M. Profiling of Arabidopsis secondary metabolites by capillary liquid chromatography coupled to electrospray ionization quadrupole time-of-flight mass spectrometry. Plant Physiol. 2004, 134, 548-559.

63. Kim, D.Y.; Jin, J.Y.; Alejandro, S.; Martinoia, E.; Lee, Y. Overexpression of AtABCG36 improves drought and salt stress resistance in Arabidopsis. Physiol Plant. 2010, 139, 170-180. [CrossRef] [PubMed]

64. Moons, A. Ospdr9, which encodes a PDR-type ABC transporter, is induced by heavy metals, hypoxic stress and redox perturbations in rice roots. FEBS Lett. 2003, 553, 370-376. [CrossRef]

(C) 2020 by the authors. Licensee MDPI, Basel, Switzerland. This article is an open access article distributed under the terms and conditions of the Creative Commons Attribution (CC BY) license (http://creativecommons.org/licenses/by/4.0/). 